\title{
Computer-controlled cylindrical polishing process for development of grazing incidence optics for the hard $\mathrm{X}$-ray region
}

\author{
Gufran S Khan $^{* a}$, Mikhail Gubarev ${ }^{\mathrm{b}}$, Chet Speegle ${ }^{\mathrm{c}}$, Brian Ramsey ${ }^{\mathrm{b}}$ \\ ${ }^{a}$ NASA Postdoctoral Program Fellow, MSFC, Huntsville, AL 35805 \\ ${ }^{\mathrm{b}}$ Space Science Office, NASA/MSFC Huntsville, AL 35805 \\ ${ }^{\mathrm{c} J a c o b s, ~ N A S A / M S F C ~ H u n t s v i l l e, ~ A L ~} 35812$
}

\begin{abstract}
The focusing performance of shell optics for the hard X-ray region strongly depends on their axial mid-spatialfrequency-range figure errors. This paper presents the development of a deterministic computer-controlled polishing process to minimize these axial figure errors on cylindrical shaped mandrels from which the mirror shells are replicated. A mathematical model has been developed to simulate the residual surface figure errors due to the polishing process parameters and the polishing tools used, along with their non-conformance to the mandrel. We present design considerations of a large-size polishing lap where the experimentally determined process variables have been used for optimizing the lap configuration and the machine operational parameters. Furthermore, the developed model is capable of generating a corrective polishing sequence for a known surface error profile. Practical polishing experiments have been performed to verify the model and to determine its ability to correct known axial figure errors through polishing machine control.
\end{abstract}

Keywords: X-ray optics, computer-controlled polishing, cylindrical polishing, mid-spatial-frequency errors

\section{INTRODUCTION}

Replicated grazing-incidence full-shell optics for hard X-ray telescopes are under development at the Marshall Space Flight Center ${ }^{1,2}$. The angular resolution of the mirror shells depends on the quality of the mandrel from which they are being replicated. Mid-spatial-frequency axial figure error is a dominant contributor in the error budget of the mandrels and arises from the mandrel fabrication process. This process involves electroless nickel-phosphorous coating, single point diamond turning and polishing. In order to improve the current achievable X-ray mirror resolution, a computercontrolled polishing process for deterministic mandrel figuring with good predictability and the best possible efficiency is in demand.

Computer-controlled polishing machines and mathematical models to analyze material removal characteristics have been developed for polishing spherical, aspheric and free form surfaces, where small-size tools are employed ${ }^{3-6}$. We presented our efforts in developing a polishing process using a large size polishing lap, where a mathematical model was developed to estimate the residual surface errors under a given set of operating parameter and lap configurations ${ }^{7,8}$. The study focused on establishing a relationship between the polishing process parameters and the mid-spatial-frequency error generation, which is crucial for developing better-quality mandrels. In this paper, we present further investigations on the optimization of the polishing process. The process variables, such as the material removal rate, and the shape and size of the tool's influence function have been determined from actual polishing runs on a mandrel. Using the extracted information of the process variables, a large-size polishing lap has been designed. This helps making the polishing process more deterministic.

Section 2 of this paper discusses the causes of mid-spatial-frequency error generation during the polishing operation. Non-conformance of the large-size rigid polishing lap to the specimen is one of the main reasons for generation of midspatial-frequency errors. The features of the developed mathematical model are then discussed in Section 3 . The experimental determination of the process variables is presented in Section 4 together with a discussion of design

*gufran_ks@yahoo.co.in; phone 1256 961-7595; fax $1256961-7522$ 
considerations of the polishing lap configuration. Section 6 reports on the experimental tests performed to verify the model.

\section{GENERATION OF MID-SPATIAL-FREQUENCY ERRORS}

The production of mandrels has traditionally employed rigid polishing tools. However, mid-spatial-frequency errors do often appear in the finished surface. These errors arise because of the inherent structure of the lap i.e. the shape and distribution of tools and grooves on the lap and the polishing process parameters, as well as non-conformance between the surface and the hard polishing pad.

\subsection{Polishing process parameters}

When a periodic structure on the lap moves back and forth on a rotating mandrel, it produces very complex mid-spatialfrequencies. The controlling process parameters are the size and shape of the individual tool on the lap, the tool-togroove ratio, and the spatial distribution of the tools over the lap surface, the length of the axial stroke, and the axial and rotational speeds of the lap and the surface respectively. These parameters will control the contour path of the individual tools over the surface. Under the assumption of constant pressure and constant relative surface speed between the tool and the workpiece, the material removal depends only on the dwell position and time.

\subsection{Non-conformance of polishing lap to the specimen}

Non-conformance between the polishing lap and the optical surface is one of the main reasons for the development of mid-spatial-frequency errors. Non-conformance arises is several ways, as discussed below:

\subsubsection{Mismatch of the shape of lap to the mandrel during stroke}

For true cylindrical surfaces, the polishing lap remains conformed to the optical surface during the whole polishing stroke. However, if the surface to be polished is conic instead of a straight cylinder, the rigid lap cannot remain conformed to the curved/tapered surface during the whole polishing stroke. As a result, contact pressure between the tool and the workpiece becomes a dynamically changing function of time and position on the workpiece. This results in a significant non-conformance error, the magnitude of which is dependent on the amount of taper in the mandrel.

\subsubsection{Existing surface error profile}

In practice, the specimen to be polished has some existing surface error to start with. Traditionally, large size pitch polishing laps are prepared by conforming them to the existing surface to be polished. The errors on the surface are thus transferred to the polishing lap during its preparation and the subsequent back and forth motion of the lap introduces non-conformance between the local topography of the surface and the polishing lap.

\subsubsection{Non-uniform compliance of lap and the mandrel}

At the commencement of polishing some of the individual tools in the lap do not have intimate contact with the specimen. After some time of polishing, they gradually become in contact as pitch starts flowing due to the friction and thermal effects. However, the non-uniform compliance during this time of polishing introduces local pressure variations leading to localized errors on the surface.

\section{MATHEMATICAL MODELING OF THE CYLINDRICAL POLISHING PROCESS}

Simulation software has been developed to predict the residual surface figure errors on a mandrel in the presence of lap non-conformance. This simulation software enables us to establish a relationship between the polishing process parameters and the mid-spatial-frequency error generation, which is crucial for developing better-quality mandrels.

The model comprises the following three features

\subsection{Residual mid-spatial-frequency error predictions}

The first mode of the model predicts the residual mid-spatial-frequency errors that depend on the process parameters. The process parameters modeled are the speeds of the lap and the mandrel, the tool's influence function, the contour path (dwell) of the tools, their shape and the distribution of the tools on the polishing lap. This mode of the software has the 
capability to compute the optical performance of the mandrel from its axial error profile. This ability to simulate the polishing process permits an understanding of the dominant sources of mid-spatial-frequency errors.

\subsection{Optimization of process parameters and polishing lap configuration}

The second mode works as an optimization routine. The optimization routine suggests a set of operating parameters and polishing lap configuration that delivers a minimum residual error in the mid-spatial-frequency range, as determined by the resulting mandrel figure half power diameter.

\subsection{Surface-error-based polishing sequence}

The third mode of the software is surface-error-based polishing sequence generation mode. It accepts an existing error profile and suggests a polishing sequence, where the lap configuration and the optimum process parameters are suggested to remove the existing axial figure errors.

\section{DETERMINATION OF PROCESS VARIABLES}

A knowledge of material removal characteristics is necessary to be able to make better predictions through our model. For this, two process variables needed to be determined; the first is the material removal rate, and the second is the tool's influence function. Material removal rate is the amount of material removed per unit time (in vol. or in depth) under a known amount of pressure/weight. The influence function represents a distribution of the material removal rate across the polishing tool and this has a characteristic shape and degree of symmetry. For example, if the leading edge of a tool removes more material than the trailing edge, the influence function becomes asymmetric in shape.

Experiments were designed to determine the material removal rate and the tool's influence function under a set of known polishing parameters such as the applied weight, the stroke length of the polishing lap, the rotational speed of the mandrel, the tool distribution on the lap, and the duration of polishing time. To gauge removal, the specimen was measured on a vertical long scan trace profilometer before and after polishing runs. To acquire the precise figure of the specimen, it was measured at four meridians 90 degrees apart. The average of the four meridians was considered a representative profile.

\subsection{Determination of material removal rate}

To extract the material removal rate, one half of the specimen was polished for two hours under a set of known operational parameters while the other half remained unpolished. Diamond-shaped tools were selected for the polishing lap configuration. Figure 1 shows the difference between the measurements before and after the polishing. The measured depth of material removed during the polishing operation was approximately 0.5 microns which makes the material removal rate $0.004 \mathrm{~m} / \mathrm{min}$. This measurement is repeated for each different set of polishing conditions.

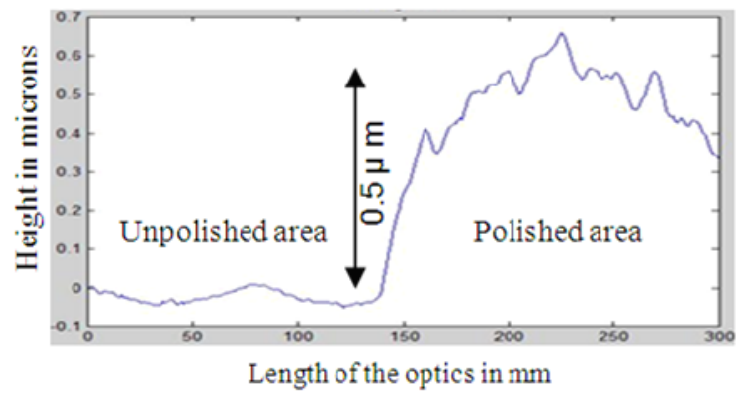

Fig.1. Difference between height measurements before and after the polishing (before - after).

\subsection{Determination of tool influence function}

The influence function has been determined by performing a known set of polishing operation on a surface with known errors. A polishing lap comprised of four square-shaped tools was prepared as shown in Figure 2a. The tools had enough separation between them so that their polishing contours on the specimen did not overlap and allowed us to be able to 
measure the amount of material removed by an individual tool. The polishing was performed for two hours with a stroke length of $30 \mathrm{~mm}$. Figure $2 \mathrm{~b}$ shows the difference between the measurements before and after the polishing run compared with simulation predictions when a step influence function is considered. The average material removed for the four tools is shown in Figure 3a. It is clear that the assumed step influence function does not deliver a good match with the amount of material removed experimentally by the four square-shaped tools. Therefore, the influence function has been back calculated to fit with the experimental material removal profile as shown in Figure $3 \mathrm{~b}$.

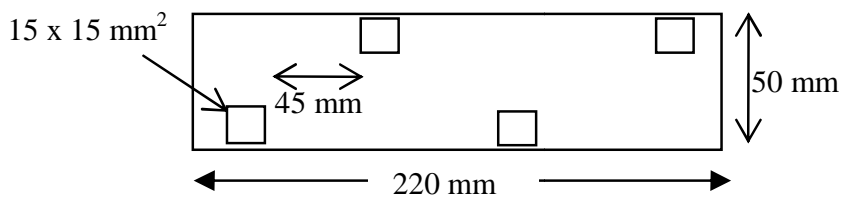

(a)

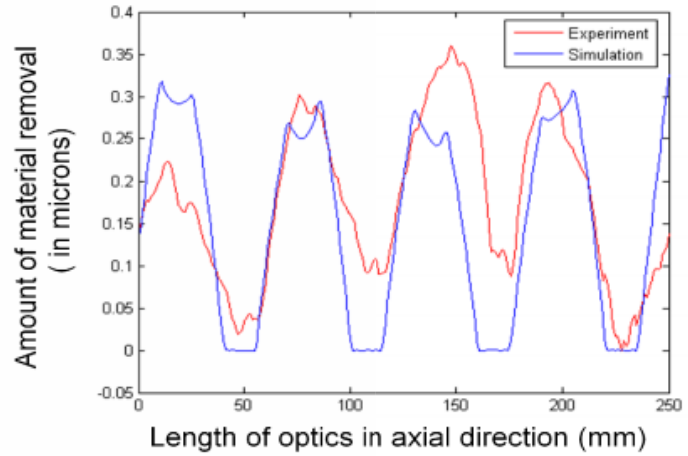

(b)

Fig. 2. a) Schematic of the polishing lap with four square-shaped tools used during the polishing run for determination of the influence function and, (b) Difference between height measurements before and after polishing.

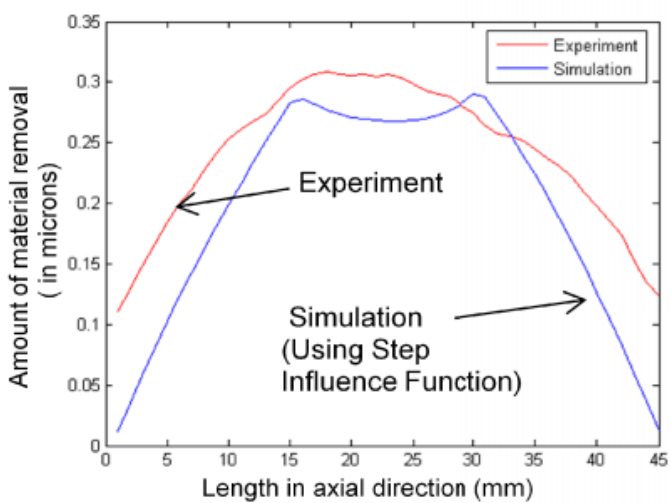

(a)

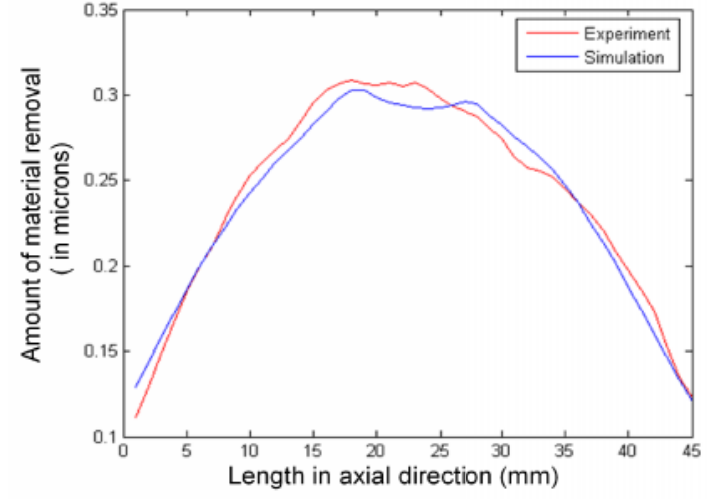

(b)

Fig. 3. a) Average profile of the material removed by four square-shaped tools, (b) Match between the simulated and experimental profile of the material removed while extracting the actual influnce function.

Figure 4 shows the shape of the extracted influence function for the square tools in the axial and azimuthal directions respectively. It is observed that the influence functions are not symmetric in shape in the azimuthal direction. We believe that the rotational directionality of the mandrel is the main reason for this asymmetry.

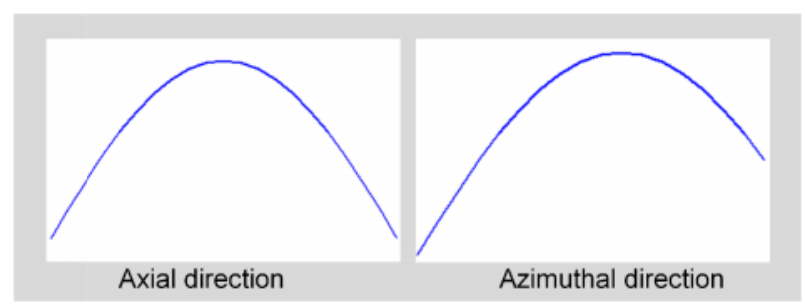

Fig. 4. Shape of the experimental influence function in the axial and azimuthal directions for the square tools. 


\subsection{Selection of polishing lap configuration}

The experimentally-measured values have been fed in to the simulation software to get an optimized design for the polishing lap and the machine operational parameters. Three types of polishing configurations, shown in Figure 5, were investigated. The figure of merit used to assess these simulated runs is the mandrel figure HPD. Figure 6 compares the performance with varying stroke length for all the three lap configurations. It was observed that equal tool-to-groove ratio with two rows of shifted tool configuration (Fig.5.b) delivered the least residual mid-spatial-frequency deviations. The optimum stroke length was found to be $30 \mathrm{~mm}$.

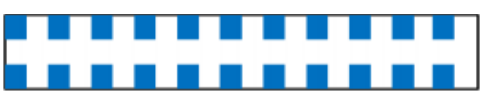

(a)

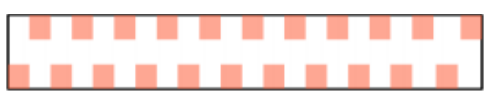

(b)

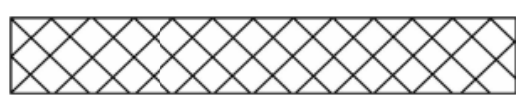

(c)

Fig. 5. Polishing lap configuration using (a) Un-shifted square tools configuration, (b) Shifted square tool configuration, (c) Diamond tools.

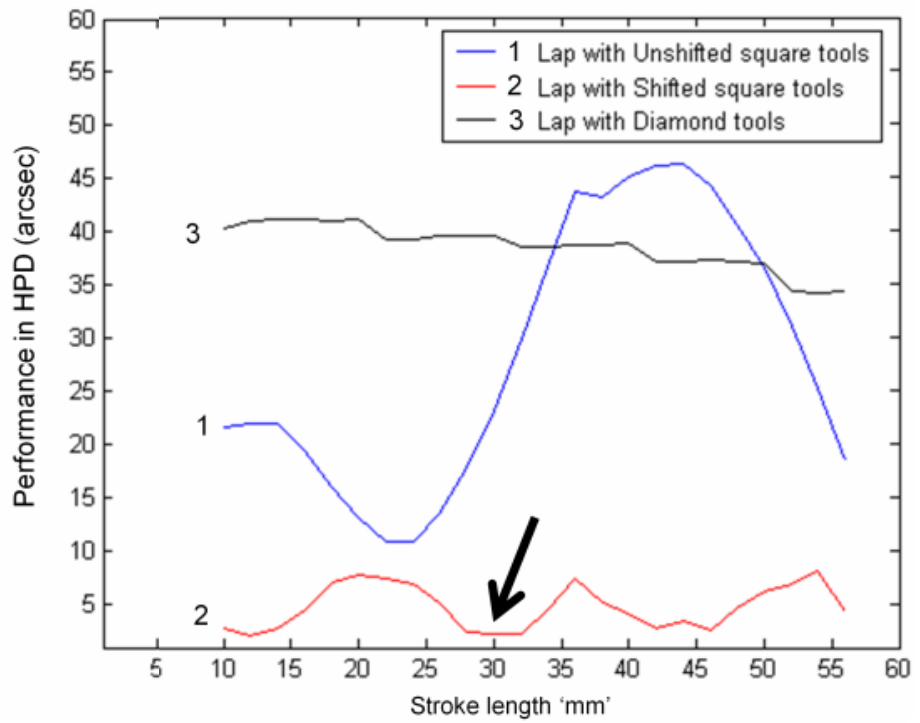

Fig. 6. Achievable performance in HPD vs stroke length in the case of (1)Un-shifted square tools configuration, (2) Shifted square tools configuration, (3) Diamond tools configuration.

\section{RESULTS AND DISCUSSION}

\subsection{Verification of the mathematical model}

A series of polishing tests were conducted to verify the predictions of the mathematical model. Using inputs from this model, a mandrel having a conical-approximated Wolter-1 geometry has been polished using the computer-controlled cylindrical polishing machine, recently developed at MSFC. Figure 7 compares the amount of material removed during actual polishing with that predicted.

The experimental results agree with the predictions in broad terms but there are disagreements in localized regions. The profiles agree in terms of the generation of frequencies but there is variation in terms of their amplitudes. At present we believe that they arise due to the non-uniformity in polishing lap compliance. Certain tools or areas of tools are either not in contact with the surface or are not applying the same pressure on the surface. Investigations are in progress to determine the level of non-uniformity. This information will also be fed into the simulations to be able make more accurate predictions. 


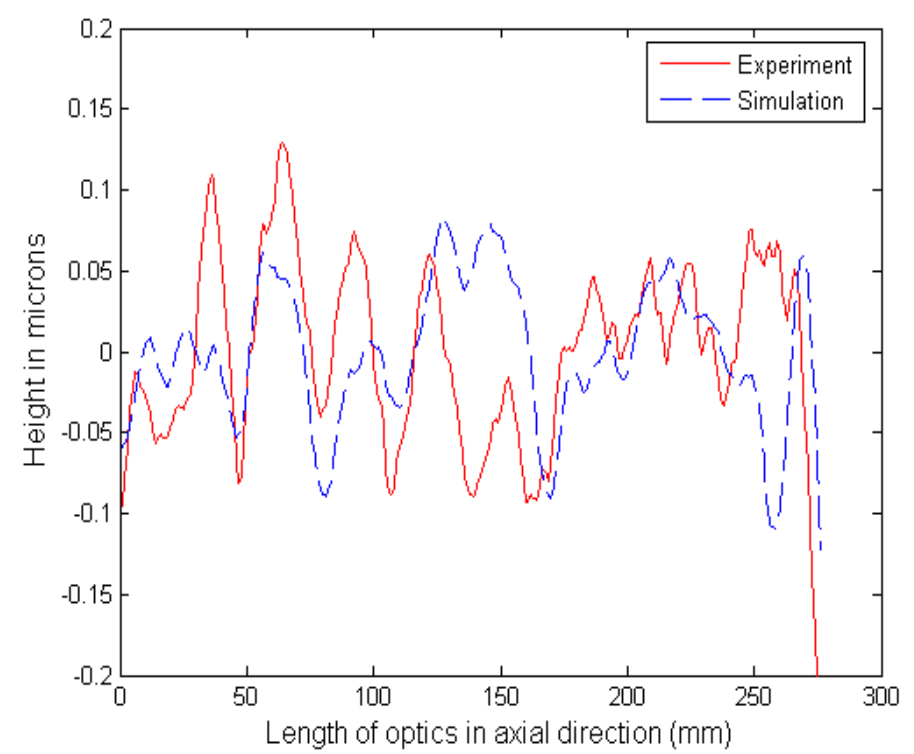

Fig. 7. Difference between height measurements before and after polishing.

\subsection{Generation and testing of surface error dependent polishing sequence}

Based on the surface error profile of the mandrel and the prior knowledge about the material removal characteristics of the polishing tool, a polishing sequence has been generated and tested for one specific example. This test involves an investigation of the density distribution of tools on the polishing lap. Three iterative polishing experimental cycles, totaling 2 machine hrs of polishing, were performed. The goal of the experiment was to determine how well and how quickly the selected profile could be corrected. As shown in Figure 8, with 2 hrs of total polishing time the surface is taken from a profile with peak-to-valley deviation of 0.85 microns to a surface having peak-to-valley deviation of 0.2 microns. The starting surface had a predicted performance of 10 arcsec while the final surface had a predicted performance of 6 arcsec half power diameter. One can still see some residual mid-spatial-frequencies present on the surface. In future, a few more error-based polishing sequences will be generated and experimentally implemented. It is expected that those polishing iterations will further improve the surface figure.

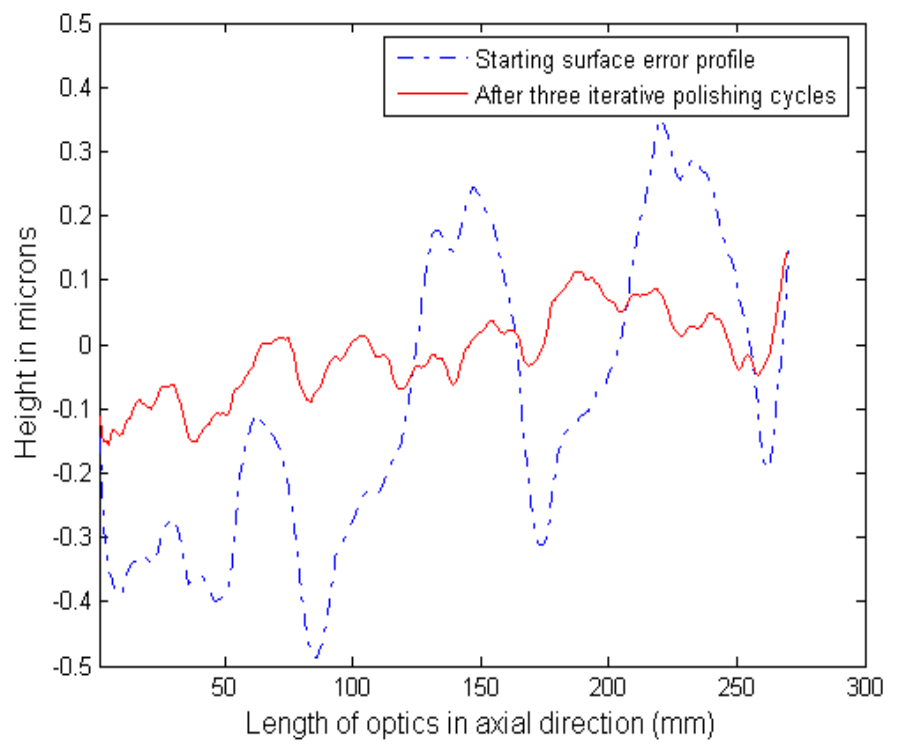

Fig. 8. Surface axial profiles; before and after three iterative surface-error-based polishing cycles. 


\section{CONCLUSIONS}

We have developed a computer-controlled polishing process using a large-size polishing lap where experimentally determined process variables have been used for optimising the lap configuration and the machine operational parameters. The experimental results have been found to be in qualitative agreement with the predictions of the simulated model. Determinism and predictability enhances the precision in polishing and at the same time makes the whole process cost effective. The model is capable of generating polishing sequences to correct a known surface error profiles. Three such polishing experiments have been performed and the results are encouraging. The information thus extracted will be used in the future for developing software for generating polishing sequences for any surface error profile.

One of the goals of developing the polishing model is to find out the achievable limits in terms of angular resolution of the replicated optics being developed at the Marshall Space Flight Center, NASA. Additionally, the ability to simulate the polishing process is an important contribution to extend automation further and thus increase the cost effectiveness of mandrel production

\section{ACKNOWLEDGEMENTS}

This research was supported by an appointment to the NASA Postdoctoral Program at the Marshall Space Flight Center, administered by Oak Ridge Associated Universities through a contract with NASA.

\section{REFERENCES}

[1] Ramsey, B. D., "Replicated nickel optics for the hard-x-ray region,” Experimental Astronomy 20, 85-92 (2005).

[2] Gubarev, M., Ramsey, B., Engelhaupt, D., and Arnold, W., "Technology Development for Nickel X-Ray Optics Enhancement," Proc. SPIE 7011, 7701133-7 (2008).

[3] Schinhaerl, M., Rascher, R., Stamp, R., Smith, L., Smith, G., Sperber, P., and Pitschke, E., "Utilisation of timevariant functions in the computer controlled polishing," Prec. Eng. 32, 47-54 (2008).

[4] Walker, D. D., Brooks, D., King, A., Freeman, R., Morton, R., McCavana, G., and Kim, S-W., "The 'Precessions' tooling for polishing and figuring flat, spherical and aspheric surfaces," Optics Express 11, 958-964 (2003).

[5] Tuell, M. T., Burge, J. H., "Aspheric optics: smoothing the ripples with semi-flexible tools," Opt. Eng. 41, 14731474 (2002).

[6] Schinhaerl, M., Smith, G., Stamp, R., Rascher, R., Smith, L., Pitschte, E., Sperber, P., and Geiss, A., "Mathematical modeling of influence functions in computer-controlled polishing: Part I," Applied Mathematical Modelling 32, 2888-2906 (2008).

[7] Khan, G. S., Gubarev, M., Arnold W., and Ramsey, B., "Development of a Computer-Controlled Polishing Process for X-Ray Optics”, Proc SPIE 7437, 74371(2009).

[8] Khan, G.S., Gubarev, M., Speegle, C., and Ramsey, B., "Deterministic Computer-Controlled Polishing Process for High-Energy X-Ray Optics," in Optical Fabrication and Testing, OSA Technical Digest (CD) (Optical Society of America, 2010), paper OThB2 (2010). 


\title{
Computer-controlled cylindrical polishing process for development of grazing incidence optics for hard X-ray region
}

by

\section{Gufran Sayeed Khan}

\author{
NASA Postdoctoral Fellow, \\ $X$-Ray Astronomy Group \\ MSFC \\ Huntsville, AL 35805, USA
}

Co-authors

Mikhail Gubarev

Chet Speegle

Brian Ramsey 


\section{Design Considerations of Polishing Lap for Computer- Controlled Cylindrical Polishing Process}

\section{OUTLINE}

- Grazing incidence X-Ray optics (...Replicated Ni Optics )

- Motivation and challenges

- Mid spatial frequency generation in cylindrical polishing

- Design considerations for polishing lap

- Simulation studies and experimental results

- Future scope

- Summary 


\section{Grazing incidence X-Ray imaging optics}

Grazing incidence optics provide high resolution imaging at X-ray energy levels

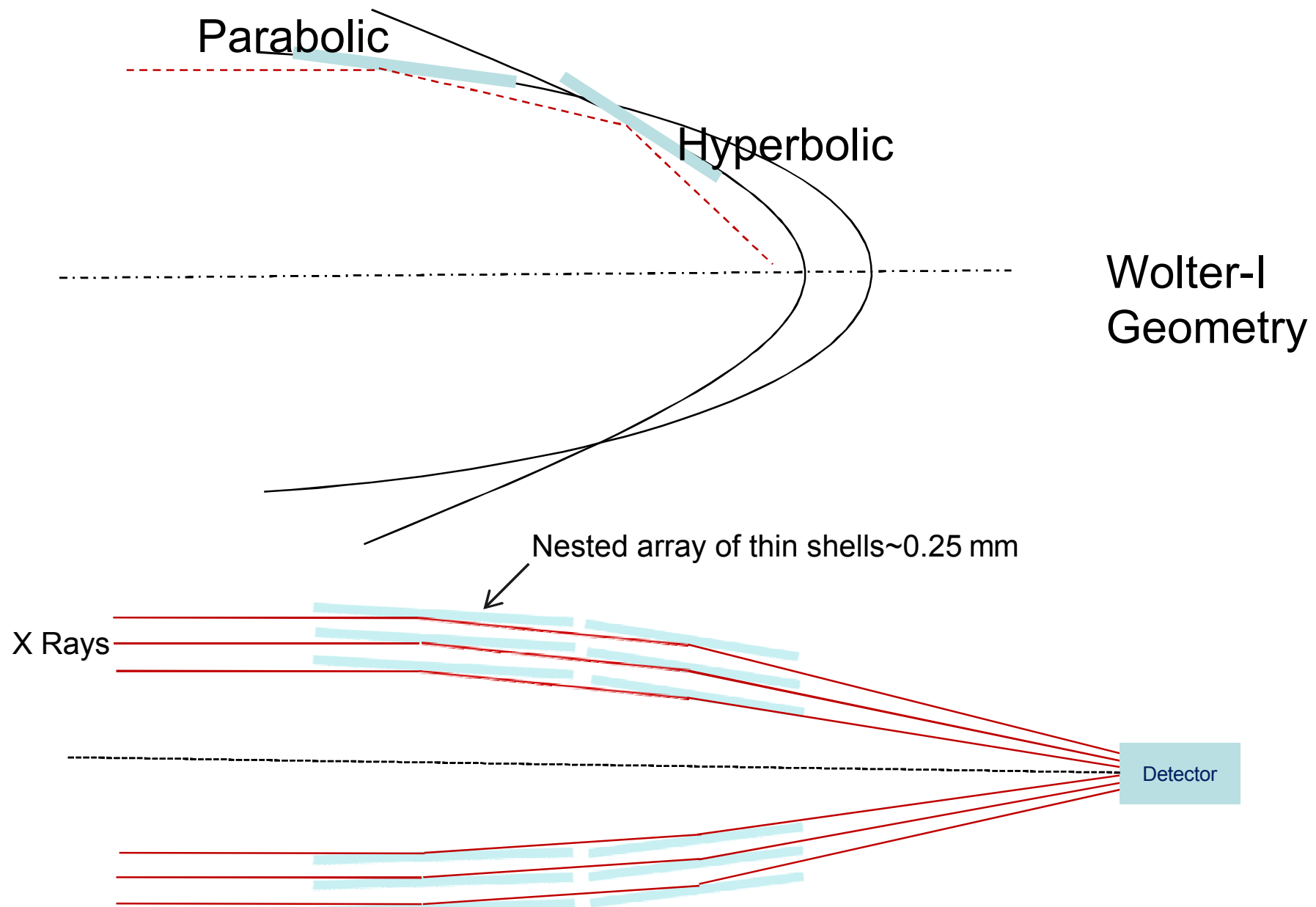

Shells are being developed by using replication approach (Electroforming) 


\section{Current status of replication optics technology}

- Typical mirror shells fabricated at MSFC

angular resolution 13-15 arcsec HPD

Mandrel $\sim 10$ arcsec HPD

- Future optics requirement

- Quality of the mandrel is the limiting factor

$\longrightarrow$ angular resolution $<5$ arcsec HPD Mandrel $\sim 2$ arcsec HPD

- Mid-spatial-frequency range errors are dominant source of error on mandrel

\section{Mid-spatial-frequency errors on mandrel}

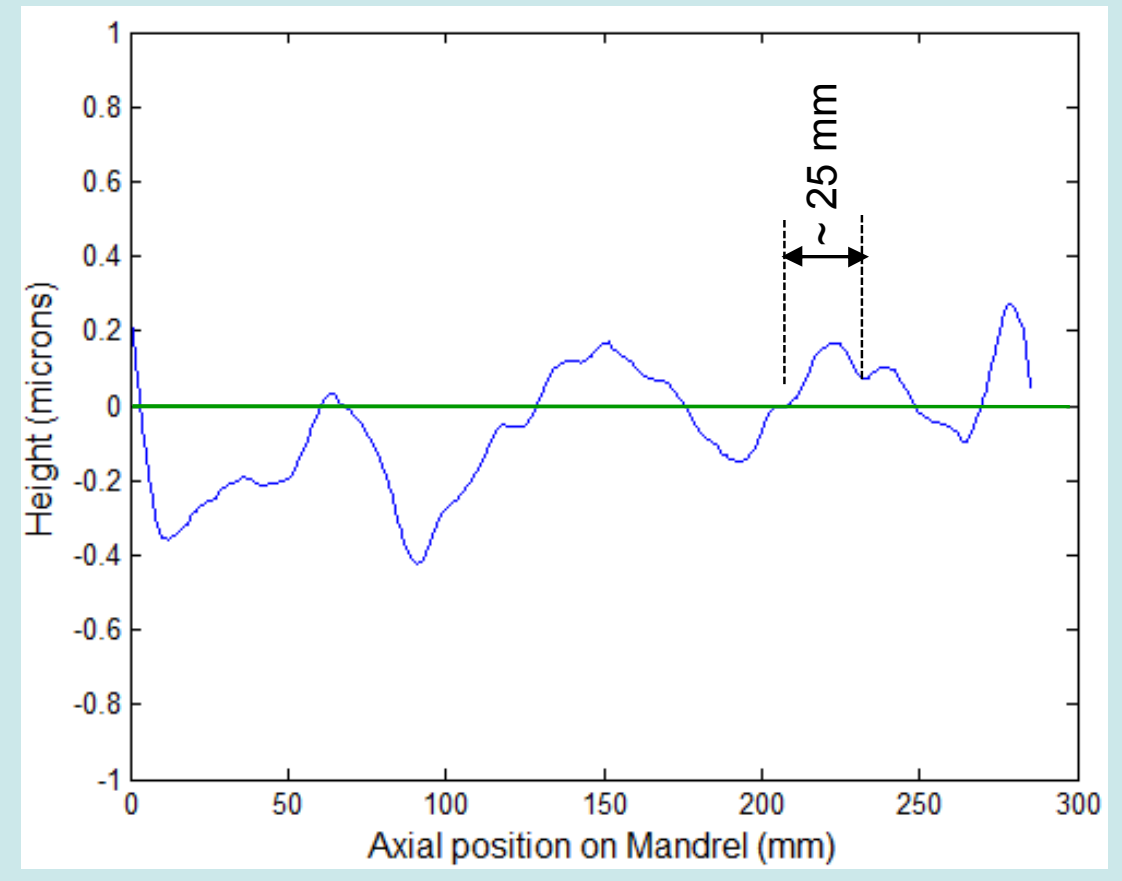

- These are 1-50 $\mathrm{mm}$ size (wavelength)

- Typically the amplitude of these errors are of $\sim 100 \mathrm{~nm}$ scale

- Mid-frequency errors are inherent to the polishing process 


\section{Polishing process}

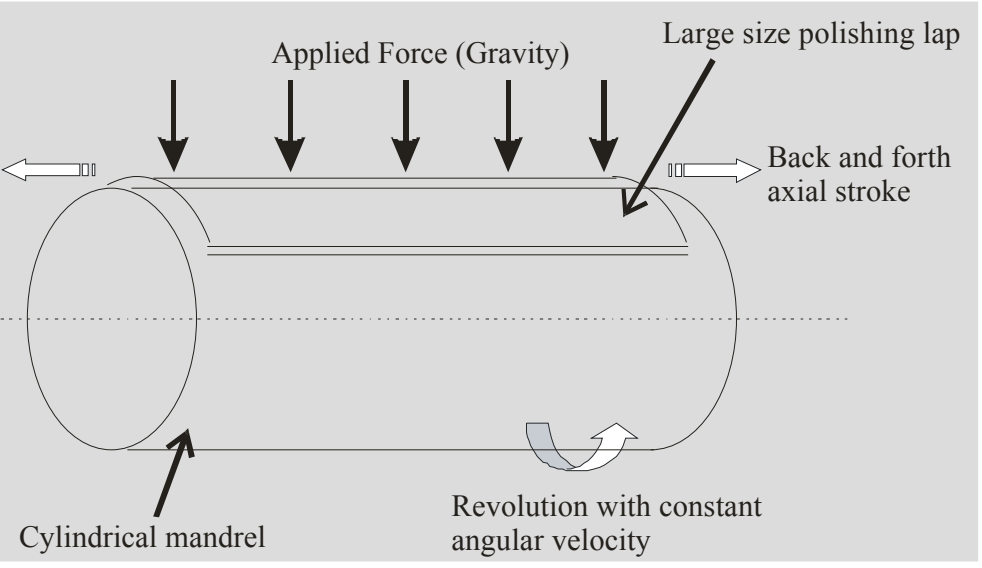

Polishing lap configuration

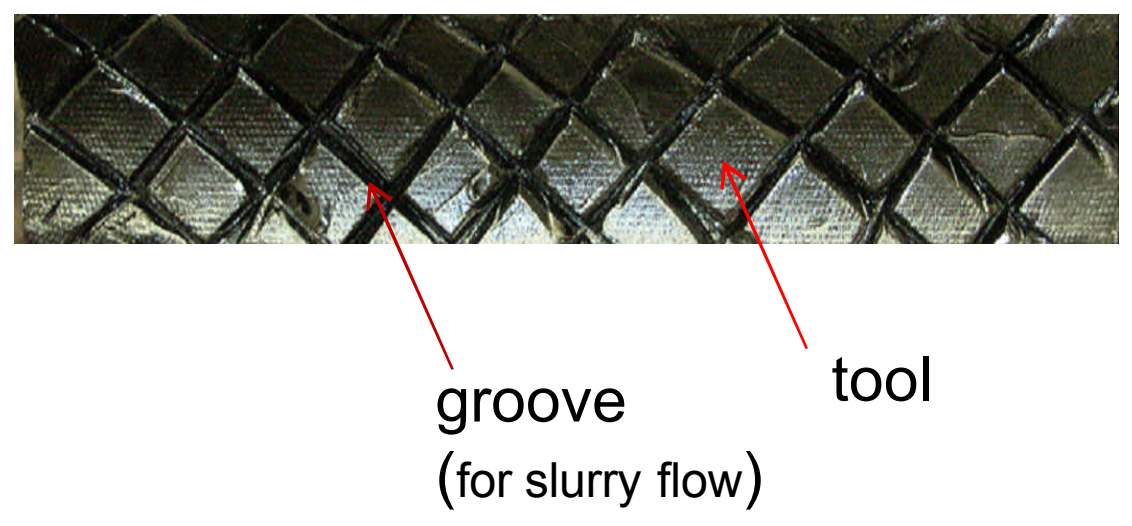

Mid-spatial-frequency range errors generation

Operating parameters

Lap configuration

- Mandrel rotational speed

- Tool size

- Lap axial speed

- Stroke length
- Tool shape

- Tool-to-groove ratio

- Distribution of tools
Non-conformance of lap to the specimen during polishing

- Pressure variation between the tool and the specimen 


\section{Non-conformance of polishing lap to the optics (Mandrel)}

(A) Non-conformance due to the mismatch of the shape of lap to the mandrel during stroke

\section{Depends on}

- Design of the optics

- Width of the polishing lap

- Stroke length during polishing

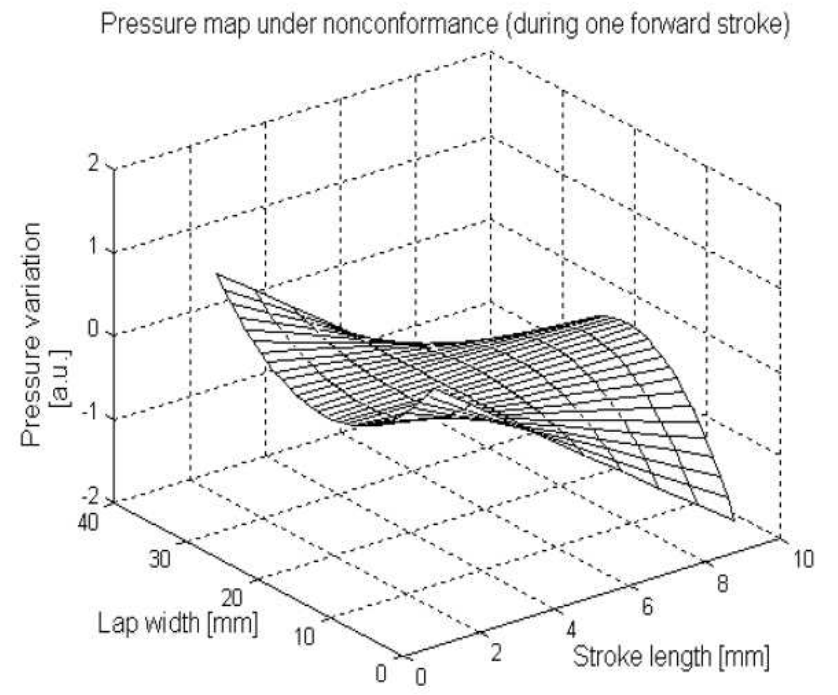

(B) Non-conformance due to the surface error profile

Depends on

Amplitude and the slope error of surface profile

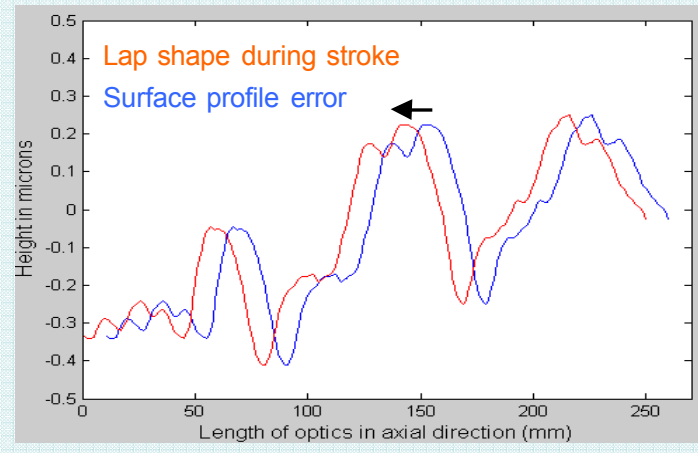

(C) Non-conformance during lap preparation (Practical non-conformance)

Depends on

Environmental effects 


\section{Development of Software and Polishing Machine}

- Developed a software to simulate cylindrical polishing process

Establishes a relationship between the polishing process parameters and the generation of mid-spatial-frequency deviations

Optimization of process parameters and lap configuration to keep the residual mid-frequency deviations to a minimum

Computer-controlled polishing machine developed at NSSTC

Mandrel

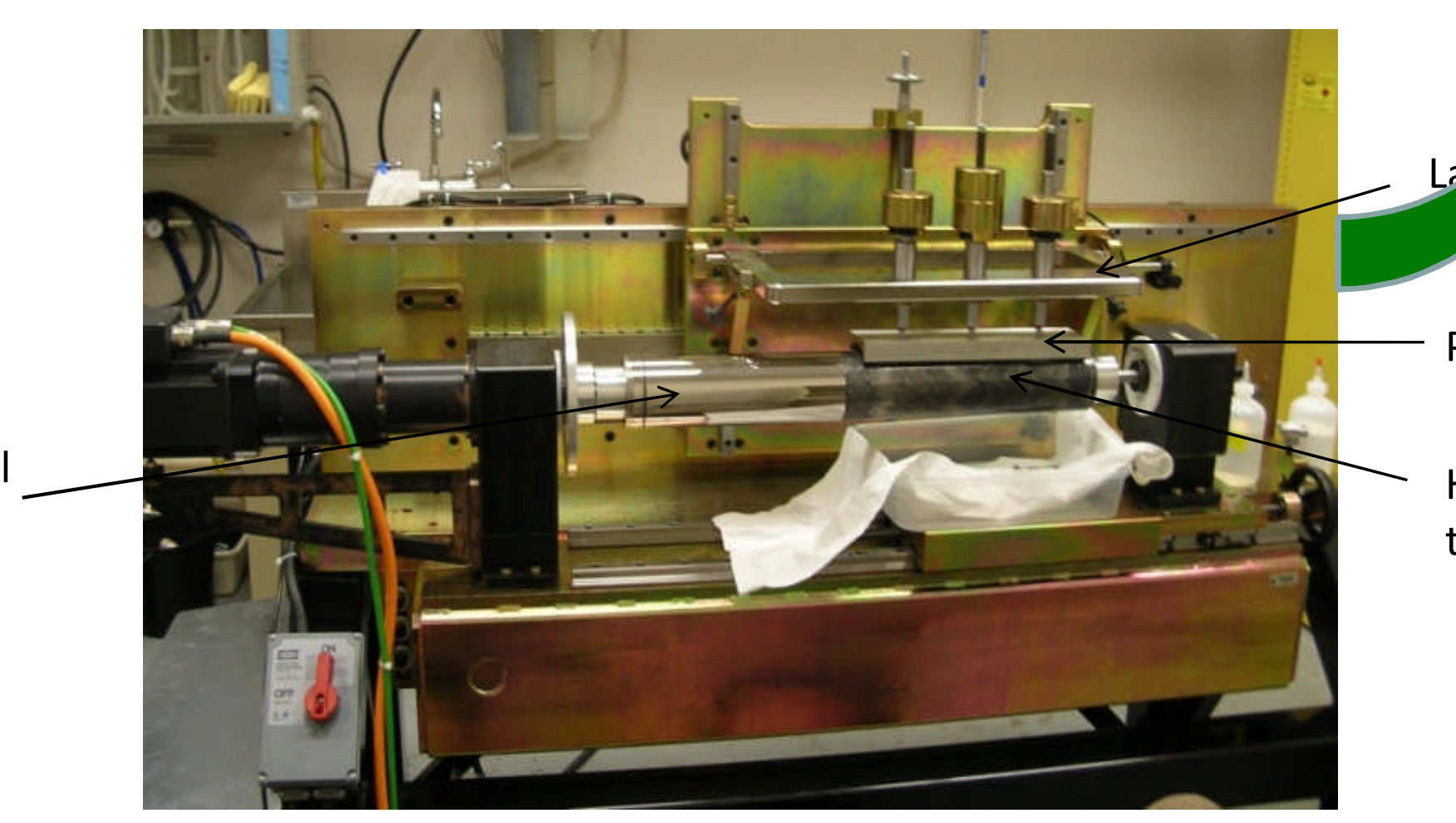
the Mandrel 


\section{A. Determination of material removal rate}

Amount of material removed per unit time (in vol. or in depth) under a known amount of pressure/weight

B. Determination of tool's influence function

Removal characteristics of the polishing tools

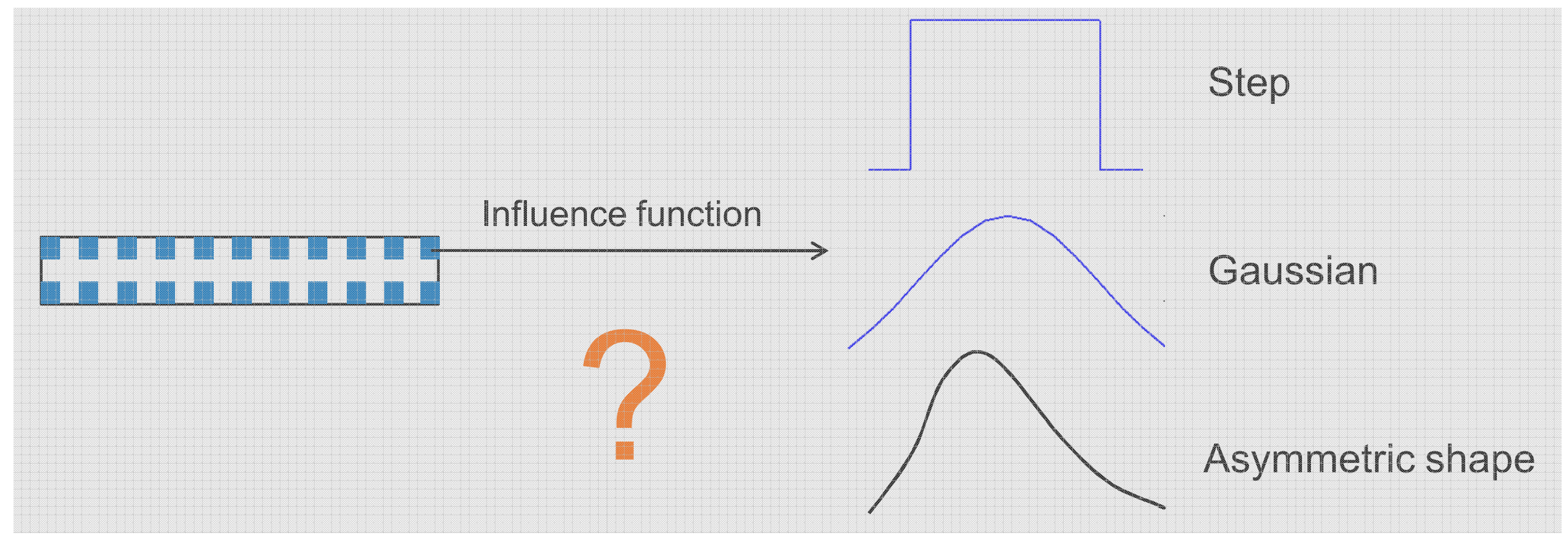

C. Selection of lap configuration and stroke length

Shape, size and distribution of tools

Optimum stroke length 


\section{A. Determination of material removal rate}

Amount of material removed per unit time (in vol. or in depth) under a known amount of pressure/weight

$>$ One half of the hyperbolic section of the mandrel was polished

$>$ Known set of operating parameters

$>$ Polishing time $\sim 2$ hrs

$>$ Diamond shape tools on the polishing lap

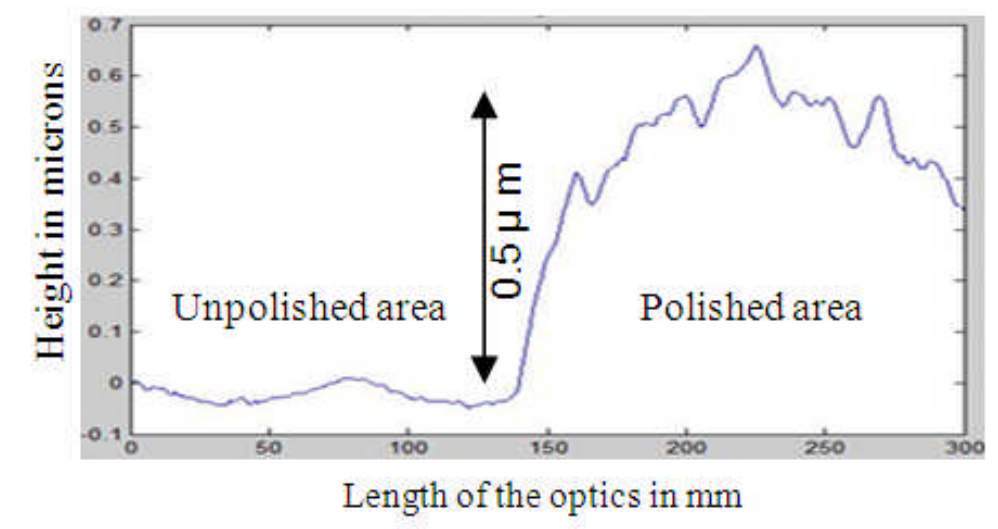

Material removal rate $0.004 \mu \mathrm{m} / \mathrm{min}$. 


\section{A. Determination of material removal rate}

B. Determination of tool's influence function
$15 \times 15 \mathrm{~mm}^{2}$
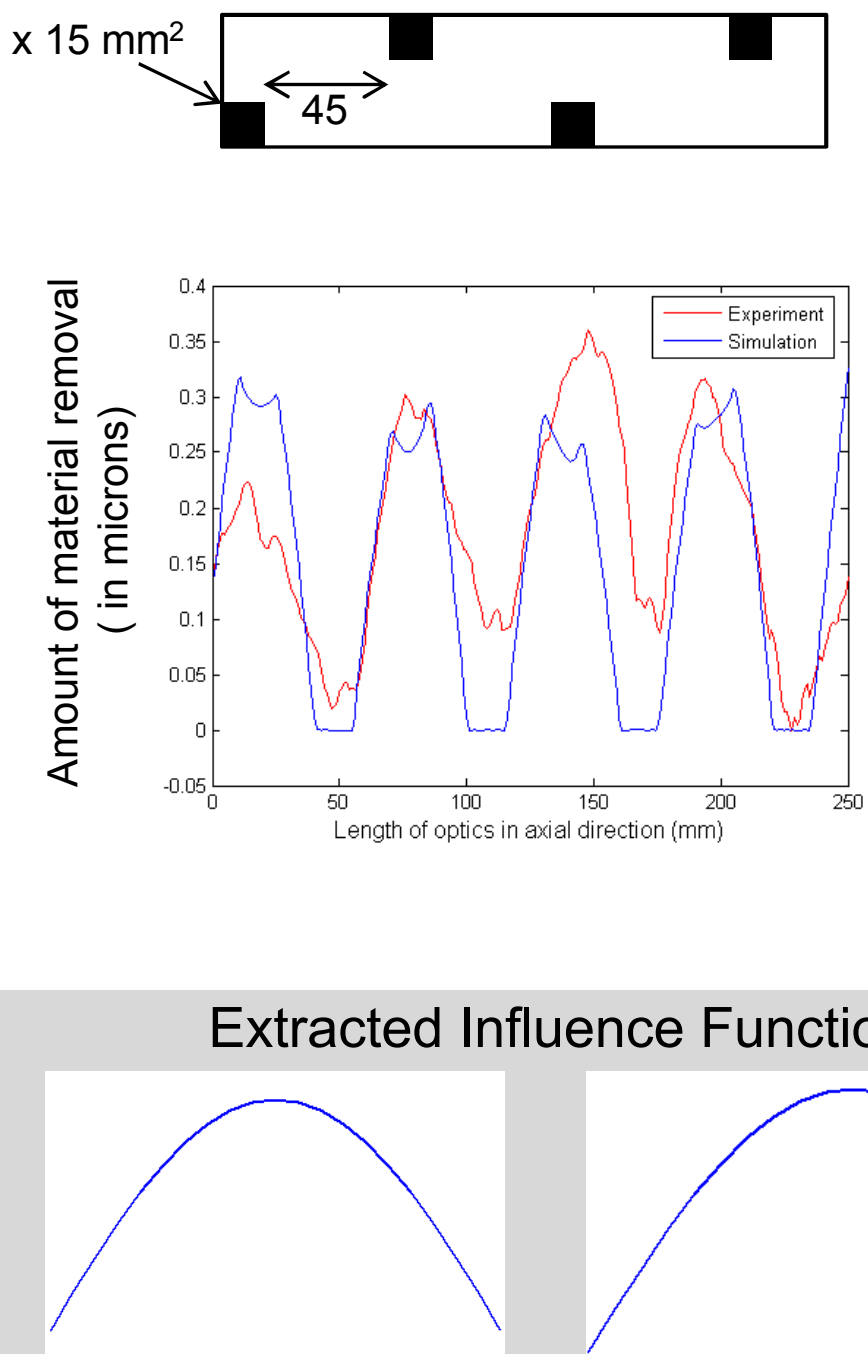

Axial direction

\section{$0.004 \mu \mathrm{m} / \mathrm{min}$.}

Simulations are performed with step influence function

Stroke length $30 \mathrm{~mm}$

Average
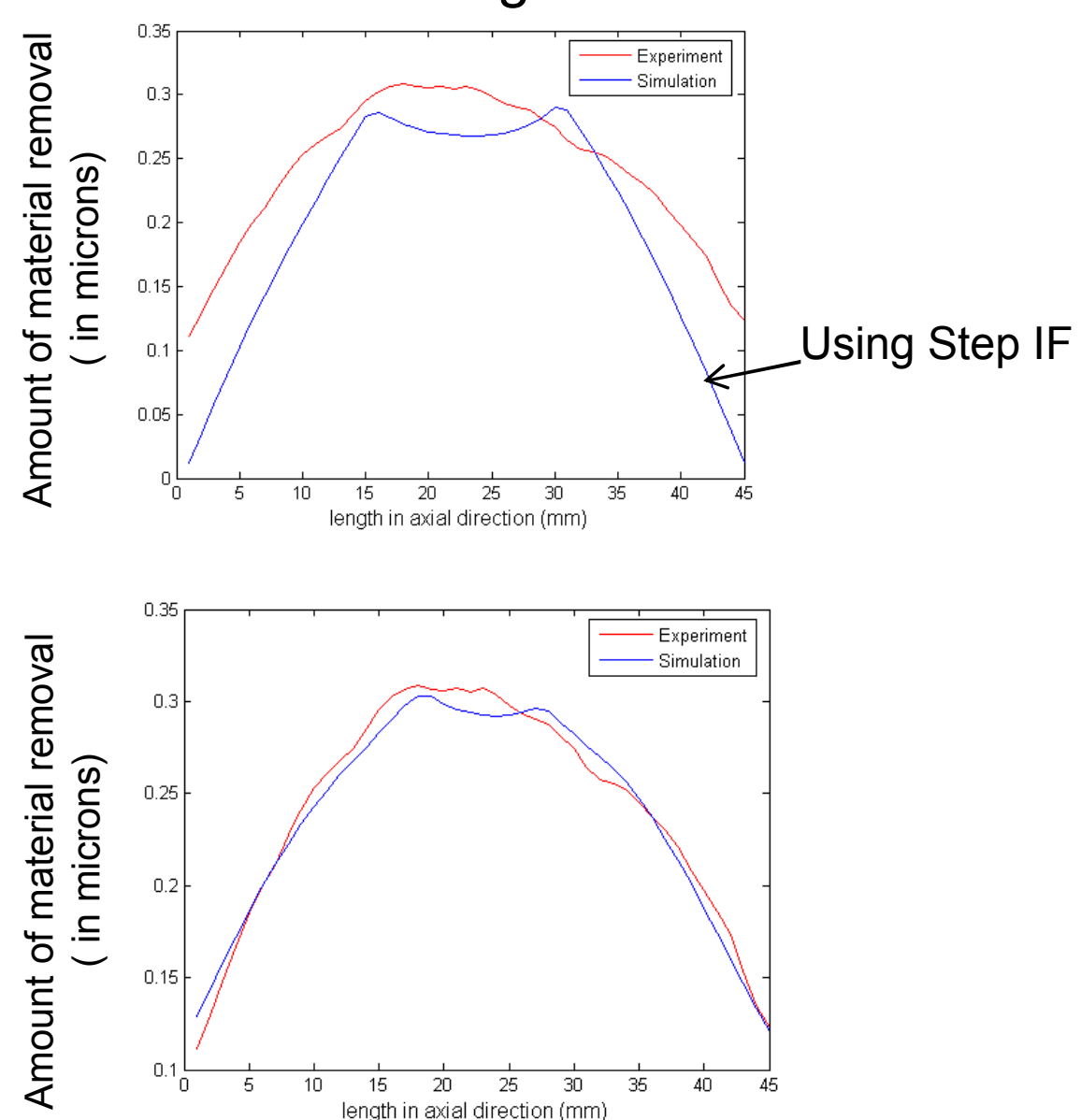
A. Determination of material removal rate

B. Determination of tool's influence function

\section{$0.004 \mu \mathrm{m} / \mathrm{min}$.}

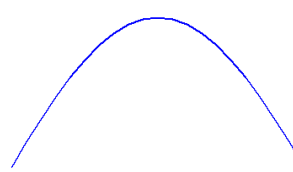

Axial direction

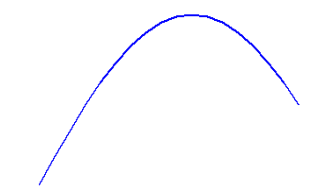

Azimuthal direction

C. Selection of lap configuration and stroke length

[Simulations]

FHAFIIIII

Un-shifted square tools

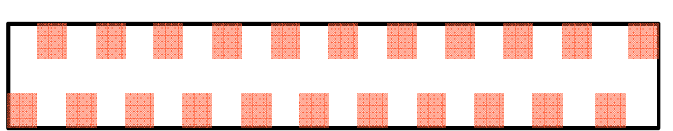

Shifted square tools

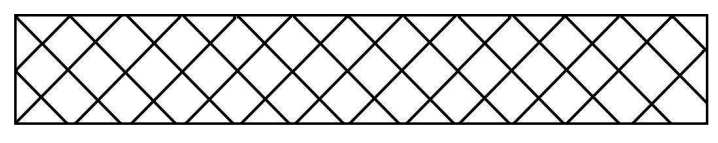

Diamond tools

\section{Performance Prediction}

Residual profile error

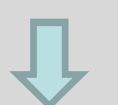

Slope error

Performance in terms of Half Power Diameter

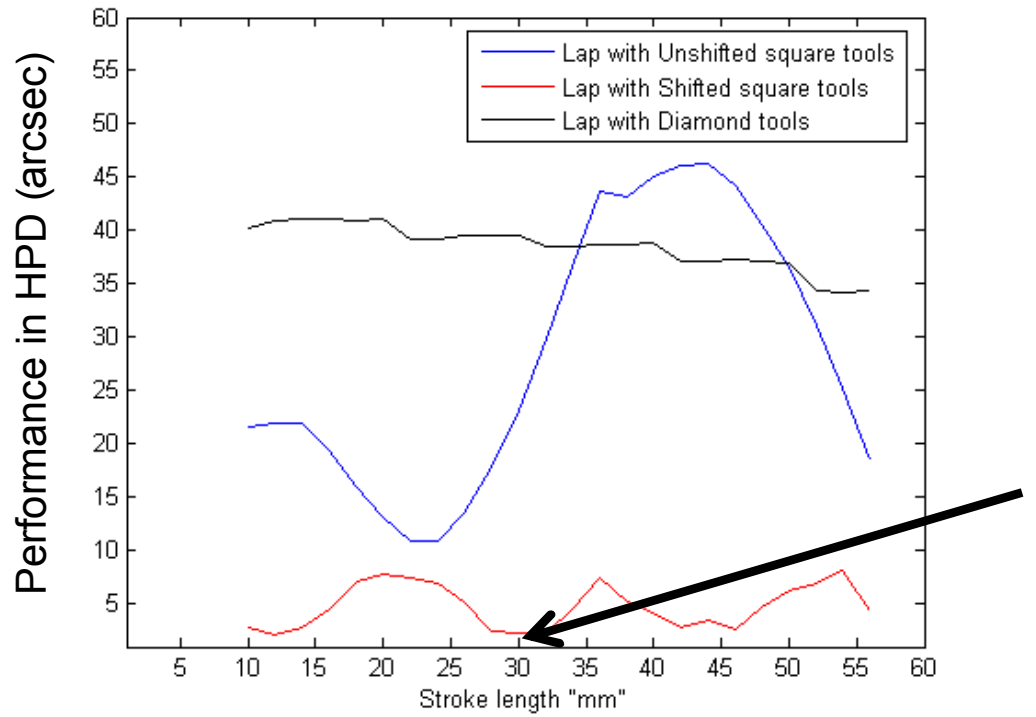

Stroke length ' $m$ ' 


\section{Deterministic prediction of polishing}

A. Determination of material removal rate

B. Determination of tool's influence function

C. Selection of lap configuration

D. Stroke length

\section{$0.004 \mu \mathrm{m} / \mathrm{min}$}

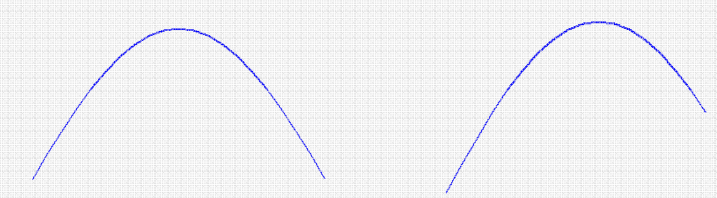

Axial direction Azimuthal direction

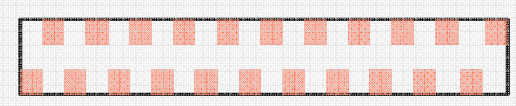

Shifted square tools

$30 \mathrm{~mm}$ 
Experiment

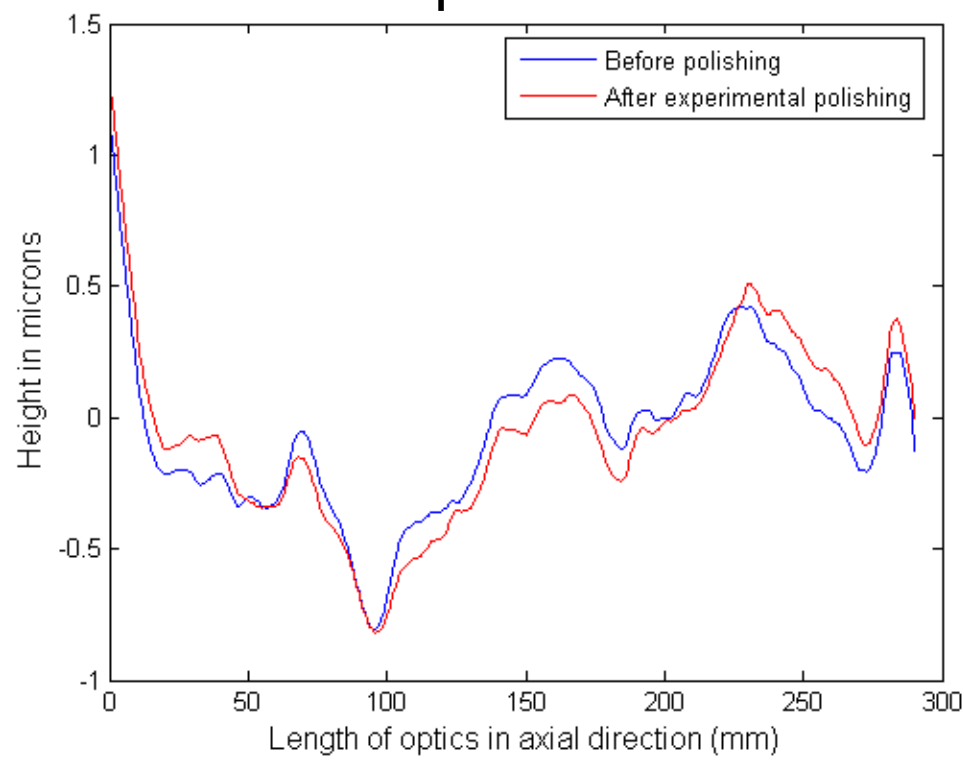

Comparison with Simulations

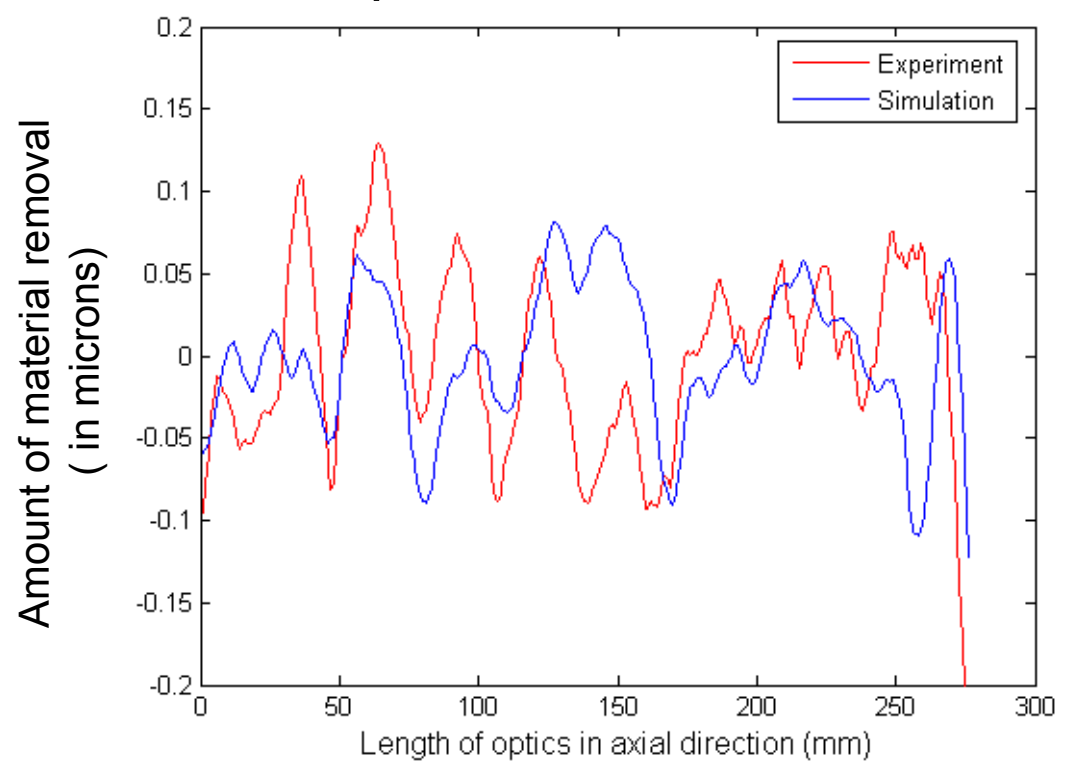

Difference (Before polishing-After polishing)

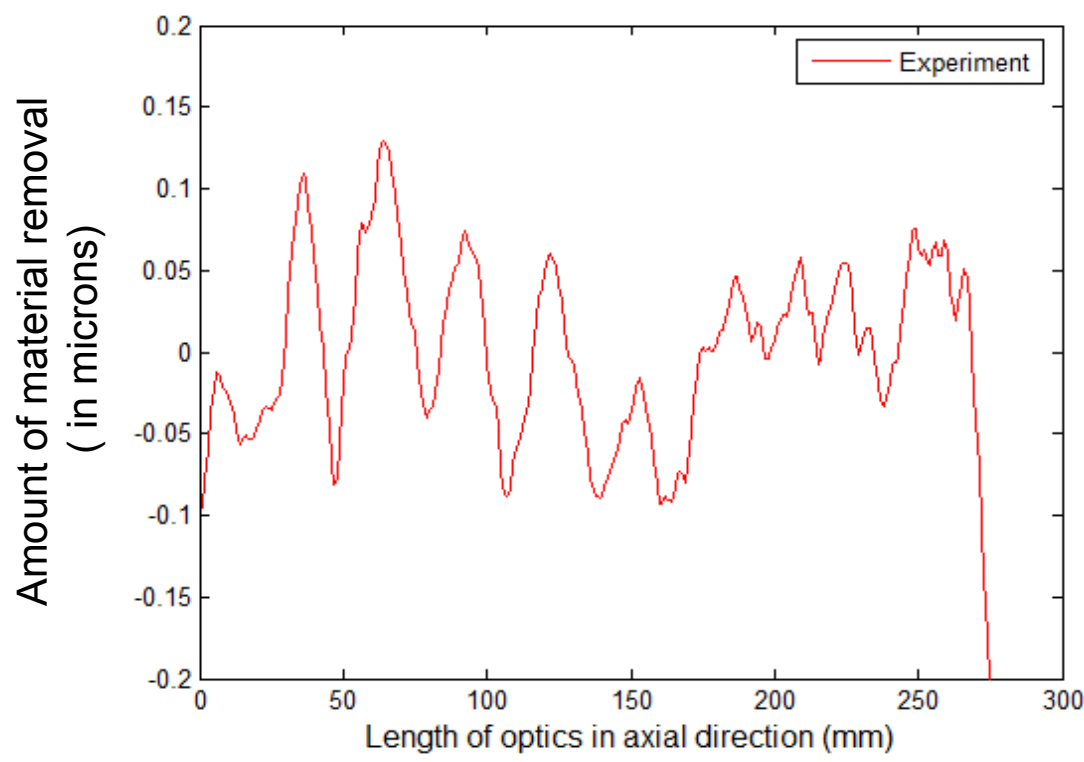

Few months back

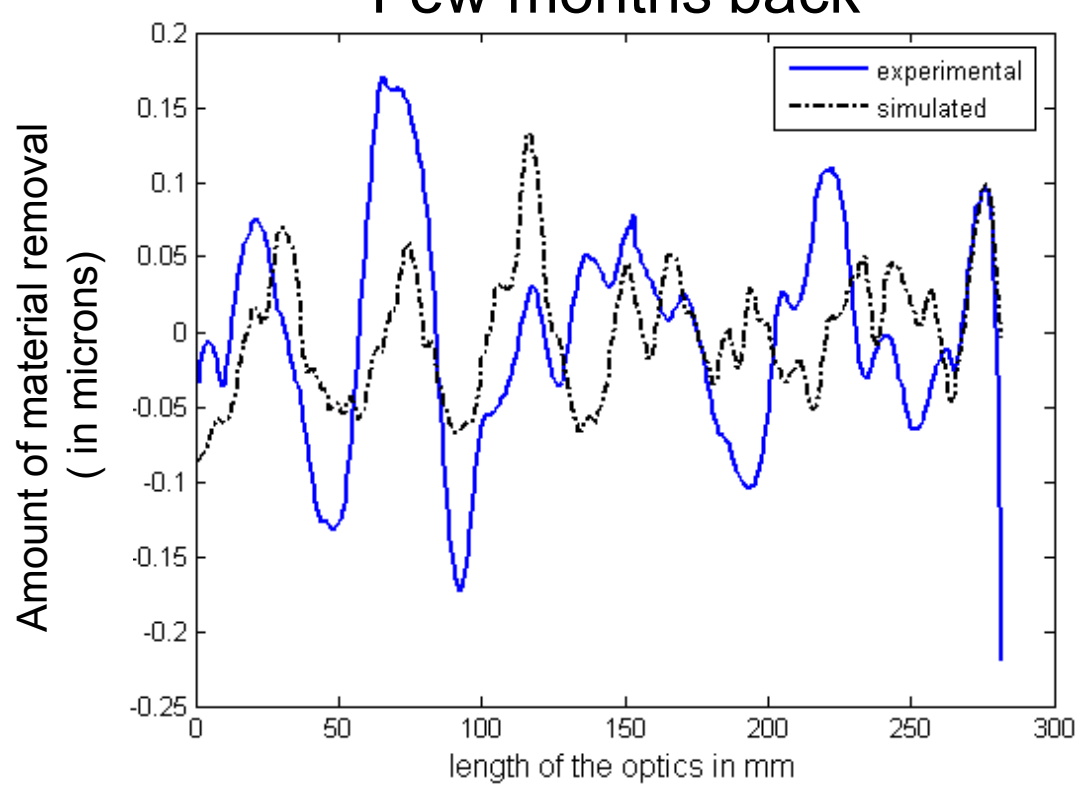


Polishing experiment based on known error profile

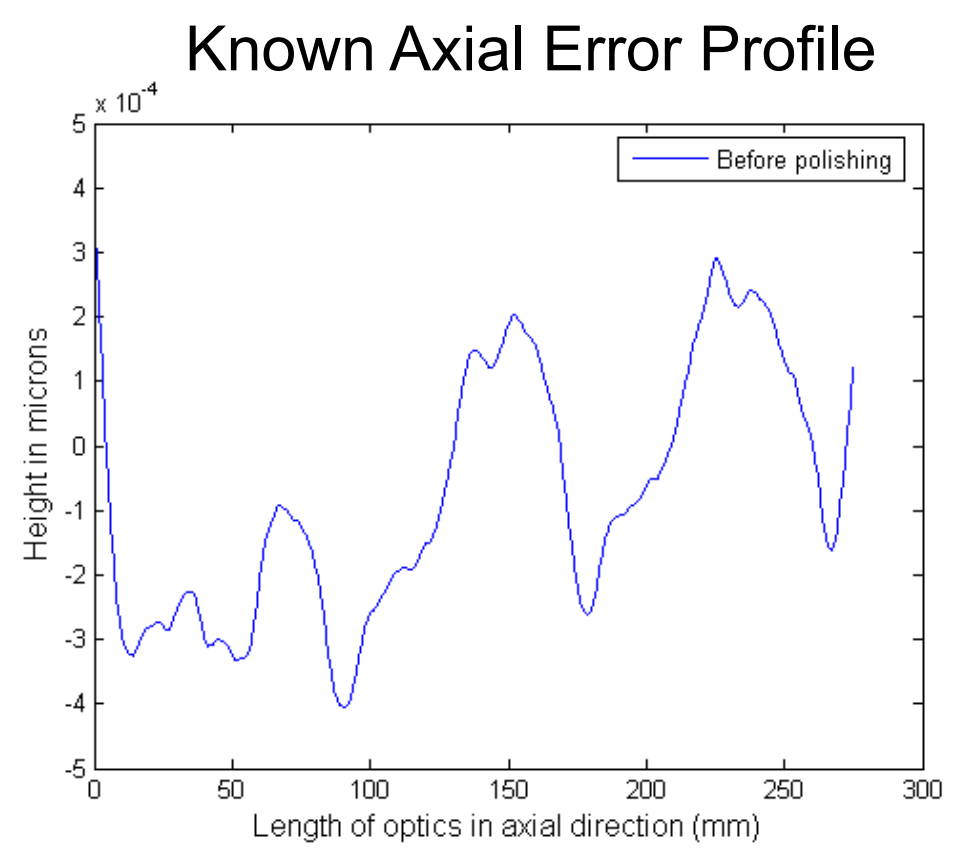




\section{Polishing experiment based on known error profile}
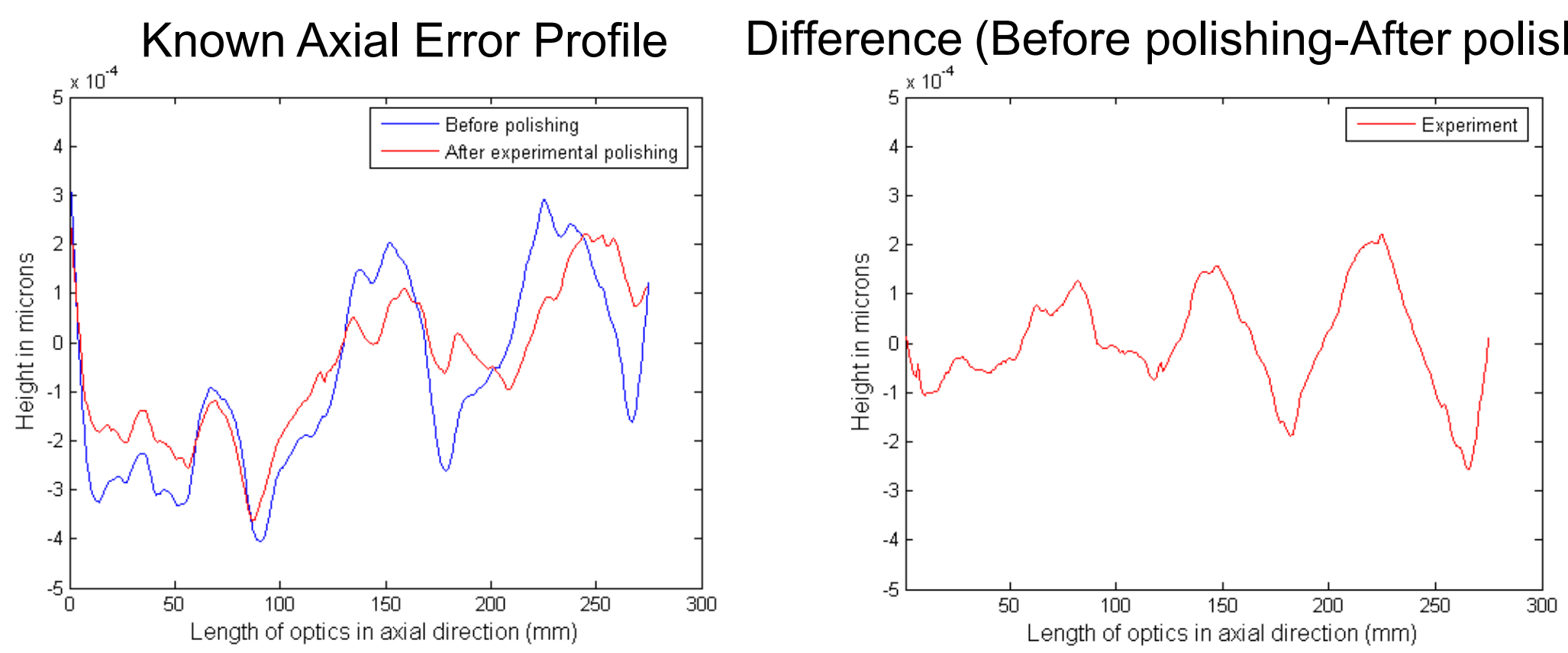

Comparison with Simulations

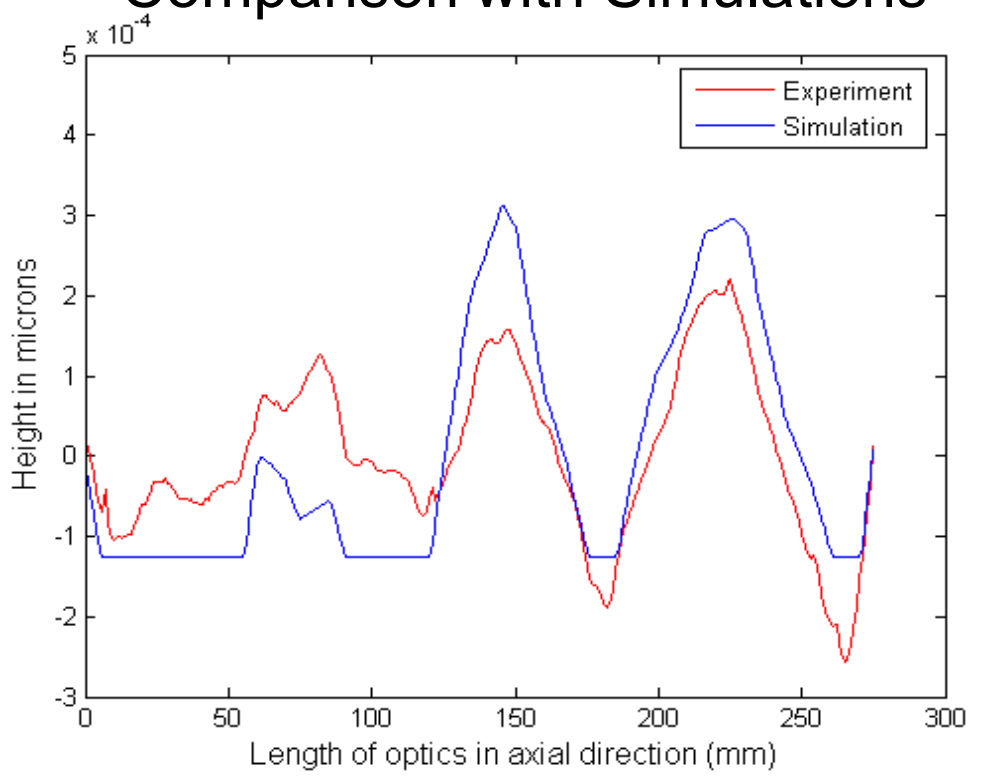




\section{Future plans}

- Determination of non-uniformity in the polishing lap compliance

- Development of a polishing sequence based on a known error profile of the specimen...... demonstration of the approach

- Software for generating a mandrel polishing sequence

- Design and development of a flexible polishing lap

- Computer controlled localized polishing process 
Thank you 